Bundesgesundheitsbl 2011 · 55:129-140 DOI 10.1007/s00103-011-1403-1

Online publiziert: 24. Dezember 2011

(c) Springer-Verlag 2011

\section{L.E. Kroll · T. Lampert}

FG 27, Abteilung für Epidemiologie und

Gesundheitsberichterstattung, Robert Koch-Institut, Berlin

\title{
Regionalisierung von Gesundheitsindikatoren
}

\section{Ergebnisse aus der GEDA-Studie 2009}

In Deutschland besteht ein zunehmender Bedarf an aktuellen und validen Daten zur gesundheitlichen Lage der Bevölkerung, dem das Robert Koch-Institut (RKI) mit der Etablierung des Gesundheitsmonitorings Rechnung getragen hat [1]. Durch das Gesundheitsmonitoring sollen der Gesundheitsberichterstattung und der Gesundheitspolitik auf Bundesebene umfangreiche Informationen zur Gesundheit, zum Gesundheitsverhalten und zur gesundheitlichen Versorgung der Bevölkerung zur Verfügung gestellt werden. Dies wird durch die drei repräsentativen Untersuchungs- beziehungsweise Interviewsurveys DEGS (Studie zur Gesundheit Erwachsener in Deutschland), GEDA (Gesundheit in Deutschland aktuell) und KiGGS (Studie zur Gesundheit von Kindern und Jugendlichen in Deutschland) gewährleistet. Alle drei Erhebungen basieren auf umfangreichen, bundesweit repräsentativen Stichproben, die es ermöglichen, Gesundheitsindikatoren für Deutschland mit einer hohen statistischen Genauigkeit auszuweisen. Eine Regionalisierung der Daten ist für den Vergleich der alten und neuen Bundesländer sowie für die bevölkerungsreichsten Bundesländer möglich.

Für viele Zusammenhänge ist es wünschenswert, nicht nur Daten auf der Bundes- oder Länderebene, sondern auch für kleinere regionale Einheiten, wie etwa Stadt- und Landkreise, verfügbar zu haben. Während in Deutschland zu regionalen Unterschieden in der Mortalität [2] oder zur Inanspruchnahme der medizinischen Versorgung [3] bereits entsprechen- de Daten zur Verfügung stehen, fehlen diese Informationen bisher für Merkmale des Gesundheitsverhaltens oder auch für die Selbsteinschätzung der Gesundheit. Eine Abschätzung der regionalen Unterschiede hinsichtlich dieser Merkmale ist beispielsweise für die Planung von Präventionsmaßnahmen von Interesse. Die Fallzahlen aus den verfügbaren Datenquellen (Health Interview oder Health Examination Surveys mit repräsentativen Stichproben) sind aber für kleinräumige Untergliederungen nicht mehr ausreichend, um über Anteilswerte hinreichend genaue und stabile Schätzwerte zu erhalten. In diesem Beitrag wird mit der sogenannten Small-Area-Estimation (SAE) ein Verfahren vorgestellt, das entsprechende Analysen ermöglichen soll [4, 5]. Das Verfahren wird anhand von Daten der GEDA-Studie 2009 exemplarisch für drei Gesundheitsindikatoren (allgemeiner Gesundheitszustand, Rauchen, Adipositas) angewendet.

Im vorliegenden Beitrag werden zunächst die Datenquellen, die verwendeten Indikatoren, die Methode der SmallArea-Estimation und die Analysestrategie beschrieben. Im Ergebnisteil wird die SAE durchgeführt und ihre Ergebnisse karto-

Tab. 1 Stichprobenbeschreibung der GEDA-Studie 2009 entlang der zentralen Untersuchungsvariablen $(n=21.262)$

\begin{tabular}{llll}
\hline & Anzahl & Anteil Stichprobe & Anteil gewichtet $^{\text {a }}$ \\
\hline Geschlecht & & & \\
\hline Männer & 9.148 & $43,0 \%$ & $48,5 \%$ \\
\hline Frauen & 12.114 & $57,0 \%$ & $51,5 \%$ \\
\hline Alter & & & \\
\hline 18-29 Jahre & 3.789 & $17,8 \%$ & $17,3 \%$ \\
\hline 30-44 Jahre & 5.976 & $28,1 \%$ & $26,2 \%$ \\
\hline 45-64 Jahre & 7.461 & $35,1 \%$ & $32,3 \%$ \\
\hline $65+$ Jahre & 4.036 & $19,0 \%$ & $24,2 \%$ \\
\hline Gesundheitszustand & & & \\
\hline Sehr gut/gut & 15.937 & $75,1 \%$ & $70,4 \%$ \\
\hline $\begin{array}{l}\text { Mittelmäßig/schlecht/ } \\
\text { sehr schlecht }\end{array}$ & 5.293 & $24,9 \%$ & $29,6 \%$ \\
\hline Aktuelles Rauchen & & & \\
\hline Nein & 15.028 & $70,7 \%$ & $70,1 \%$ \\
\hline Ja & 6.223 & $29,3 \%$ & $29,9 \%$ \\
\hline Adipositas (BMl > 30) & & & $84,0 \%$ \\
\hline Nein & 18.047 & $86,6 \%$ & $16,0 \%$ \\
\hline Ja & 2.791 & $13,4 \%$ & \\
\hline aGewichtung nach Geschlecht, Alter, Bundesland und Bildung auf die Bevölkerung Deutschlands am 31.12.2008 \\
\hline
\end{tabular}


Tab. 2 Verwendete Indikatoren auf Ebene der kreisfreien Städte und Landkreise aus der INKAR-Datenbank 2009. Quelle: [12], Bezugsjahr 2007 (sofern nicht anders vermerkt)

Indikator mit Bezugszeitrum

Aktivierungsquote bei Arbeitslosen (SGB II)

Ambulante Pflege 2005

Anteil Pflegebedürftige 2005

Arbeitslosenquote nach Geschlecht

Arztdichte (Einwohner je Arzt)

Ausländeranteil

Baulandpreise 2006/2007

Betreuungsquote Kleinkinder 15.03.2007

Betreuungsquote Vorschulkinder 15.03.2007

Bruttoinlandsprodukt je Einwohner 2006

Durchschnittlicher Rentenzahlbetrag

Einwohner nach Altersgruppen

Einwohnerdichte

Erholungsfläche je Einwohner 2004

Erwerbsquote nach Geschlecht

Freifläche je Einwohner 2004

Gesamtwanderungssaldo

Getötete im Straßenverkehr

Haushaltseinkommen 2006

Industriequote

Kinderärzte je 100.000 Kinder

Krankenhausbetten 2006

Langzeitarbeitslosenquote

Lebenserwartung nach Geschlecht

Naturnähere Fläche je Einwohner 2004

Personen je Hauhalt 01.01.2006

Pkw-Dichte

Sanktionsquote bei Arbeitslosen

SGB II - Quote

Siedlungsdichte 2004

Stationäre Pflege 2005

Straßenverkehrsunfälle

Veränderung Lebenserwartung nach Geschlecht 1995-2007

Verunglückte im Straßenverkehr

grafisch veranschaulicht. Diesen Ergebnissen werden Vergleichswerte aus dem Mikrozensus gegenübergestellt. Abschließend werden die Ergebnisse zusammengefasst und Möglichkeiten und Limitationen der Small-Area-Estimation diskutiert.

\section{Daten und Methode}

Zur Analyse des Zusammenhangs zwischen sozialräumlichen Faktoren und Gesundheitsindikatoren werden die Daten der Studie „Gesundheit in Deutschland aktuell“ (GEDA) 2009 sowie die INKARDatenbank 2009 verwendet. Die Daten der GEDA-Studie liefern dabei Individualdaten, die aufgrund der verfügbaren Angaben zum Wohnort der Befragten, auf Ebene der Landkreise und kreisfreien Städte regionalisiert werden können. Die Daten aus der INKAR-Datenbank liefern die Indikatoren zur sozialen und gesundheitlichen Lage des räumlichen Kontextes auf Kreisebene. Die Verknüpfung beider Datenquellen erfolgt dabei über Selbstangaben zum Wohnort der Studienteilnehmer.

\section{Gesundheit in Deutschland aktuell (GEDA) 2009}

Bei der GEDA-Studie 2009 handelt es sich um eine telefonische Befragung, die zwischen Juli 2008 und Juni 2009 im Rahmen des Gesundheitsmonitorings durch das Robert Koch-Institut durchgeführt wurde $[1,6]$. Die Stichprobenbasis stellt eine nach dem Gabler-Häder-Verfahren gezogene Zufallsstichprobe von Telefonnummern dar $[7,8]^{1}$. Die Grundgesamtheit der Studie bilden alle in Privathaushalten lebenden Erwachsenen, die über einen Festnetzanschluss verfügen. Die Themen der Erhebung gliedern sich in einen Kernbereich, der auch in künftigen Befragungen konstant bleiben soll, sowie in einen flexiblen Themenbereich, in den aktuelle Fragestellungen oder Themen einbezogen werden. Insgesamt wurden in der Stu-

\footnotetext{
${ }^{1}$ Durch das Verfahren, das als, „random digit dialing method" bekannt ist, wird eine repräsentative Zufallsstichprobe von Telefonnummern von Privathaushalten aus der Grundgesamtheit der in Deutschland vergebenen Festnetznummern von Privathaushalten generiert. Es handelt sich um den methodischen Standard für Telefonstichproben in Deutschland [8]. Die Ausgangsbasis bilden alle, zum Zeitpunkt der Ziehung des Samples, im Telefonbuch verzeichneten Nummern. Damit auch nicht verzeichnete Nummern (sogenannte "Geheimnummern") in die Stichprobe gelangen, werden aus den vergebenen Nummernblöcken (Orsvorwahl + Rufnummer abzüglich der letzten beiden Stellen) neue Rufnummern per Zufallsgenerator generiert. Die Nummernblöcke gehen gewichtetet nach ihrer Häufigkeit im Telefonbuch in den Generator ein. Das Nummernsample für die GEDA-Studie 2009 wurde dem RKI durch das GESIS Institut bereitgestellt.
}

die $\mathrm{n}=\mathbf{2 1 . 2 6 2}$ Personen befragt. Die Ausschöpfung auf Zielpersonenebene, also das Verhältnis zwischen abgeschlossenen Interviews und der Gesamtzahl der kontaktierten Zielpersonen, beträgt 51,2\% [9]. Die Daten der Studie lassen sich auf Basis der Selbstangaben der Befragten zu ihrem Wohnort auf Kreisebene regionalisieren. Der Wohnort ist für $\mathrm{n}=21.063 \mathrm{Be}$ fragte auf Basis von Selbstangaben dokumentiert, die mittlere Anzahl von Befragten pro Kreis in der GEDA-Studie 2009 beträgt $n=51(S D=65,0)$. Alle 413 Kreise und kreisfreien Städte in Deutschland (Stand: 01.08.2008) sind dabei vertreten.

In den Analysen soll die regionale Verteilung des selbsteingeschätzten Gesundheitszustandes, der Rauchquote und der Prävalenz von Adipositas für Deutschland untersucht werden. Der selbsteingeschätzte Gesundheitszustand hat sich in vielen Studien unabhängig von medizinisch objektivierbaren Indikatoren als guter Prädiktor für die Lebenserwartung und die Inanspruchnahme des Versorgungssystems erwiesen [10]. Es wird der Anteil von Personen ausgewiesen, die angeben, dass ihr Gesundheitszustand „mittelmäßig“, „schlecht“ oder „sehr schlecht“ ist. Als Raucher werden im Folgenden Personen angesehen, die im Interview angaben, aktuell regelmäßig oder zumindest gelegentlich Zigaretten, Zigarren, Pfeifen oder ein anderes Tabakprodukt zu konsumieren. Adipositas wird über Selbstangaben zu Körpergröße [m] und Körpergewicht $[\mathrm{kg}]$ erhoben. Sie beginnt nach einer durch die WHO festgelegten Klassifikation bei einem BMI über $30\left[\mathrm{~kg} / \mathrm{m}^{2}\right]$. Bei der Einordnung der Ergebnisse zur Adipositasprävalenz muss allerdings berücksichtigt werden, dass der BMI auf Basis dieser Selbstangaben im Vergleich zu Messwerten unterschätzt wird [11]. Die zentralen Kennzahlen der Indikatoren aus der GEDA-Studie 2009 sind in $\bullet$ Tab. 1 dargestellt.

\section{INKAR 2009 - Indikatoren, Karten und Grafiken zur Raum- und Stadtentwicklung in Deutschland und in Europa}

Bei der Datenbank INKAR 2009 handelt es sich um eine regelmäßig aktualisierte Zusammenstellung von Raumin- 
dikatoren, die vom Bundesinstitut für Bau-, Stadt- und Raumforschung (BBSR) - einer Ressortforschungseinrichtung des Bundesministeriums für Verkehr, Bau und Stadtentwicklung - bereitgestellt wird [12]. Die Daten sind für wissenschaftliche Zwecke zu beziehen. Die Indikatoren liegen in der Datenbank auf verschiedenen regionalen Tiefen vor. Die höchste Ebene stellen die Bundesländer, die unterste Ebene die Kreise und kreisfreien Städte dar. Die verwendete Version der Datenbank stammt - ebenso wie die Daten der GEDA-Studie - aus dem Jahr 2009. Sie enthält circa 500 Indikatoren zu den Themenbereichen Bevölkerung und Sozialstruktur, Wirtschaft und Beschäftigung, Arbeitsmarkt, Einkommen und Verdienst, Wohnen, Bildung, soziale und medizinische Versorgung, Verkehr und Erreichbarkeit, Flächennutzung und Umwelt sowie Finanzen und öffentliche Haushalte. Die regionale Gliederung auf der Ebene der Kreise entspricht dem Stand vom 01.08.2008.

Für die Analysen wurden Indikatoren mit inhaltlichem Bezug zur gesundheitlichen Lage der Bevölkerung aus der INKAR-Datenbank 2009 exportiert und den Individualdaten der GEDA-Studie 2009 über die amtliche Kreisnummer zugespielt. Die Auswahl der Indikatoren orientierte sich dabei an gängigen sozialepidemiologischen Erklärungsmodellen zu sozialen Determinanten der Gesundheit $[13,14,15,16]$. Einbezogen wurden Indikatoren zu den Bereichen Arbeitsmarkt, Arbeitslosigkeit, Wirtschaftsstruktur, Öffentliche und private Finanzen, Gesundheitliche Lage, Unfallrisiken, Struktur der medizinischen Versorgung, Bildungsindikatoren sowie Siedlungsstruktur. In den jeweiligen Bereichen wurden, sofern möglich, Indikatoren gewählt, für die auf der Individualebene eine Assoziation mit Gesundheitsoutcomes gut dokumentiert ist (beispielsweise Arbeitslosenquote und Gesundheitsrisiken von individueller Arbeitslosigkeit; Unterschiede im Gesundheitszustand zwischen den Einkommensgruppen und Haushaltseinkommen). In einigen auf der Individualebene bedeutsamen Bereichen lagen keine hinreichend passgenauen Kontextindikatoren vor, sodass Indikatoren gewählt wurden, die wahrscheinlich mit dem je-

Bundesgesundheitsbl 2011 · 55:129-140 DOI 10.1007/s00103-011-1403-1

(C) Springer-Verlag 2011

\section{L.E. Kroll·T. Lampert \\ Regionalisierung von Gesundheitsindikatoren. Ergebnisse aus der GEDA-Studie 2009}

\section{Zusammenfassung}

Die bundesweit repräsentativen Gesundheitssurveys des Robert Koch-Instituts wurden bisher nicht herangezogen, um Aussagen zu regionalen Unterschieden in der gesundheitlichen Lage zu machen. Mit der "Small-Area-Estimation" wird ein Verfahren beschrieben, das es künftig ermöglichen soll, zu kleinräumigen Schätzwerten für Gesundheitsindikatoren zu gelangen. Im vorliegenden Beitrag werden anhand dieses Verfahrens mit den Daten der Studie "Gesundheit in Deutschland aktuell 2009" regionale Unterschiede auf Kreisebene für den allgemeinen Gesundheitszustand („nicht gut" oder "sehr gut"), die Rauchquote und die Prävalenz von Adipositas analysiert. Dazu werden den Daten der GEDA-Studie Regionalindikatoren aus der INKAR-Datenbank 2009 zu- gespielt. Die Ergebnisse werden mit thematischen Karten grafisch dargestellt. Anschließend werden die Ergebnisse den Daten aus dem Mikrozensus 2005 des Statistischen Bundesamtes gegenübergestellt. Dabei zeigt sich eine große Übereinstimmung beider Datenquellen. Die Small-Area-Estimation hat somit das Potenzial, zukünftig kleinräumige epidemiologische Daten für die Gesundheitsberichterstattung bereitzustellen. Dadurch könnte in Zukunft etwa die Evidenz für die Planung von Präventionsmaßnahmen weiter verbessert werden.

\section{Schlüsselwörter}

Regionale Unterschiede · Mehrebenenanalysen - Gesundheitsberichterstattung · Rauchen $\cdot$ Adipositas

\section{Regionalization of health indicators. Results from the GEDA-Study 2009}

\section{Abstract}

The representative health surveys conducted by the Department of Epidemiology and Health Reporting weren't used before to provide estimates for the spatial distribution of health outcomes. We are discussing the possibilities of providing these outcomes using methods for 'Small-Area-Estimation'. In the study we are using data of the "German Health Update 2009" (GEDA) to analyze regional inequalities for self-assessed health status, smoking and obesity on the district level in Germany. The small area estimates are provided by multilevel logistic regression models using additional regional statistical data from the official INKAR 2009 database of regional indicators for Germany. We are mapping the results of our analysis for the district level (NUTS-3) using simple thematic maps. Afterwards we compared the results of our small area models with conventional estimates that were based on the official German small scale census. The results showed that our estimates are in line with the prevalences of the census. Overall the results suggest that Small-Area-Estimation methods have a big potential to provide regionalized health indicators for the health reporting in Germany.

Keywords Spatial Epidemiology · Multilevel Analysis . Health Reporting $\cdot$ Smoking $\cdot$ Obesity 
Tab. 3 Bedeutsame Einflussfaktoren auf die Prävalenz der drei Gesundheitsindikatoren nach Kontrolle für Geschlecht, Alter und Haushaltsgröße. Datenbasis: GEDA 2009, INKAR 2009

\begin{tabular}{|c|c|c|c|c|}
\hline \multirow[t]{2}{*}{ Kontextmerkmale } & \multirow[t]{2}{*}{ Beschreibung } & \multicolumn{3}{|l|}{ Effekt } \\
\hline & & SuG & Rau & Adi \\
\hline $\begin{array}{l}\text { Arbeitslosenquote Män- } \\
\text { ner }\end{array}$ & \multirow[t]{2}{*}{$\begin{array}{l}\text { Anteil der Arbeitslosen an den Er- } \\
\text { werbspersonen in } \%\end{array}$} & & • & \\
\hline Arbeitslosenquote Frauen & & & - & \\
\hline $\begin{array}{l}\text { Einpersonen-Bedarfsge- } \\
\text { meinschaften }\end{array}$ & $\begin{array}{l}\text { Anteil der Bedarfsgemeinschaften } \\
\text { mit } 1 \text { Person an den Bedarfsgemein- } \\
\text { schaften insgesamt in \% }\end{array}$ & & • & \\
\hline Erwerbsquote Männer & $\begin{array}{l}\text { Erwerbspersonen je } 100 \text { Einwohner } \\
\text { im erwerbsfähigen Alter }\end{array}$ & & - & \\
\hline Sanktionsquote & $\begin{array}{l}\text { Anteil der erwerbsfähigen Hilfe- } \\
\text { bedürftigen mit mindestens einer } \\
\text { Sanktion an den erwerbsfähigen } \\
\text { Hilfebedürftigen in \% }\end{array}$ & • & • & \\
\hline SGB II - Quote & $\begin{array}{l}\text { Anteil der erwerbsfähigen und nicht } \\
\text { erwerbsfähigen Personen im SGB II } \\
\text { an den unter 65-Jährigen in \% }\end{array}$ & - & & \\
\hline $\begin{array}{l}\text { Betreuungsquote Klein- } \\
\text { kinder }\end{array}$ & $\begin{array}{l}\text { Anteil der Kinder unter drei Jahren } \\
\text { in Kindertageseinrichtungen an } \\
\text { den Kindern der entsprechenden } \\
\text { Altersgruppe }\end{array}$ & & - & \\
\hline $\begin{array}{l}\text { Bruttoinlandsprodukt je } \\
\text { Einwohner }\end{array}$ & $\begin{array}{l}\text { Bruttoinlandsprodukt in } 1.000 \text { Euro } \\
\text { je Einwohner }\end{array}$ & • & • & - \\
\hline $\begin{array}{l}\text { Haushaltsnettoeinkom- } \\
\text { men }\end{array}$ & $\begin{array}{l}\text { Verfügbares Einkommen der Haus- } \\
\text { halte in Euro je Einwohner }\end{array}$ & • & & \\
\hline Dienstleistungsquote & $\begin{array}{l}\text { SV Beschäftigte im Dienstleistungs- } \\
\text { sektor je } 100 \text { Einwohner im erwerbs- } \\
\text { fähigen Alter }\end{array}$ & • & • & \\
\hline Industriequote & $\begin{array}{l}\text { SV Beschäftigte in der Industrie je } \\
100 \text { Einwohner im erwerbsfähigen } \\
\text { Alter }\end{array}$ & • & & \\
\hline Lebenserwartung Männer & $\begin{array}{l}\text { Lebenserwartung von Männern bei } \\
\text { Geburt }\end{array}$ & & - & • \\
\hline Ambulante Pflege & $\begin{array}{l}\text { Anteil der Pflegebedürftigen in } \\
\text { ambulanter Pflege an den Pflegebe- } \\
\text { dürftigen insgesamt in \% }\end{array}$ & - & & \\
\hline Krankenhausbetten & $\begin{array}{l}\text { Krankenhausbetten je } 10.000 \text { Ein- } \\
\text { wohner }\end{array}$ & • & & - \\
\hline Pflegebedürftige & $\begin{array}{l}\text { Pflegebedürftige je } 10.000 \text { Ein- } \\
\text { wohner }\end{array}$ & • & & \\
\hline Einwohner je Arzt & $\ldots$ & • & & • \\
\hline Stationäre Pflege & $\begin{array}{l}\text { Anteil der Pflegebedürftigen in } \\
\text { stationärer Pflege an den Pflegebe- } \\
\text { dürftigen insgesamt in \% }\end{array}$ & & & - \\
\hline Unfalltote & $\begin{array}{l}\text { Getötete im Straßenverkehr je } \\
100.000 \text { Einwohner }\end{array}$ & & & - \\
\hline Baulandpreise & $\begin{array}{l}\text { Durchschnittliche Kaufwerte für } \\
\text { Bauland in Euro je } \mathrm{m}^{2}\end{array}$ & • & & \\
\hline Einwohnerdichte & Einwohner je km² & & • & \\
\hline
\end{tabular}

weiligen Faktor assoziiert sind (beispielsweise für Bildungsstand der Bevölkerung die Betreuungsquote von Kleinkindern, die Dienstleistungsquote und die Sied- lungsdichte). In der Literatur zur geografischen Gesundheitsforschung ist dokumentiert, dass Assoziationen zwischen Kontextmerkmalen und Gesundheitsin- dikatoren einerseits auf direkte Effekte der Kontextmerkmale, andererseits aber auf die soziodemografische Komposition der Bevölkerung in den Kontexten zurückzuführen sein können („,context versus composition" Problem) [17]. In dieser Studie kann nicht geklärt werden, ob für die verwendeten Indikatoren kontextuelle oder kompositionelle Effekte dominieren.

Alle verwendeten kreisbezogenen Indikatoren sind in $\bullet$ Tab. 2 dargestellt. Es wurde immer der aktuellste Wert des jeweiligen Indikators verwendet. Sofern nicht anders vermerkt, handelt es sich dabei um den Jahresdurchschnitt 2007. Das zentrale Kriterium bei der Auswahl von Regionalindikatoren für die nachfolgenden Analysen war die Plausibilität eines Zusammenhangs mit den untersuchten Gesundheitsoutcomes auf der Individualebene auf Basis gängiger sozialepidemiologischer Erklärungsansätze [18]. Aus vielen für die Individualebene vorliegenden Studien ist bekannt, dass in der Bevölkerung hinsichtlich aller drei Gesundheitsindikatoren beträchtliche sozioökonomische Differenzen bestehen [19, 20]. Aus der INKAR-Datenbank wurden daher neben Bevölkerungsstrukturdaten vor allem Sozialindikatoren extrahiert. Um die Prävalenzschätzungen weiter zu verbessern, wurden zusätzlich auch Indikatoren verwendet, die nicht als Determinanten der Gesundheit, sondern als Krankheitsfolgen $\mathrm{zu}$ interpretieren sind, wie zum Beispiel die Inanspruchnahme medizinischer Leistungen und die Sterblichkeit $[21,22]$.

Insgesamt wurden aus der INKAR-Datenbank 44 Indikatoren als mögliche regionale Determinanten der Gesundheitsoutcomes ausgewählt. Im Vergleich zur herkömmlichen logistischen Regression gestaltet sich die Parameterschätzung für das logistische Mehrebenenmodell mit zufälligen Effekten (Random Intercept Modelle) deutlich aufwendiger [23]. Darum konnten nicht alle vorab als relevant eingeschätzten Indikatoren in die Modelle aufgenommen werden. Vielmehr musste vorbereitend eine Auswahl von relevanten Indikatoren getroffen werden. Für das Auswahlverfahren kamen herkömmliche logistische Regressionsmodelle zum Einsatz. Ausgehend vom vollständigen Modell mit allen kontextuellen und indivi- 
duellen Determinanten wurden dabei schrittweise alle Kontextmerkmale, deren $\mathrm{p}$-Wert über $\mathrm{p}=0,20$ lag, ausgeschlossen. Diese sogenannte stepwise-Prozedur wird in der Datenanalyse kritisch eingeschätzt, da auch inhaltlich relevante Einflussfaktoren ausgeschlossen werden können [24]. Mit $p=0,20$ wurde ein „weiches" Auswahlkriterium bei der Prozedur gewählt, um die Wahrscheinlichkeit zu verringern, dass relevante Merkmale ausgeschlossen werden. Merkmale, die auf der Individualebene gemessen wurden (Alter, Geschlecht und Haushaltsgröße), sind von der Auswahlwahlprozedur ausgenommen worden. Die Berücksichtigung der Haushaltsgröße ist dabei methodisch begründet. So ist die Haushaltsgröße in Telefonumfragen - wie der hier verwendeten GEDA-Studie 2009 - mit der Auswahlwahrscheinlichkeit der Teilnehmer assoziiert. Sie wird für gewöhnlich in der Designgewichtung berücksichtigt. Da in den Mehrebenenmodellen keine Gewichtungsfaktoren einbezogen werden konnten, wurde durch die Aufnahme dieses Merkmals in das Modell für designbedingte Verzerrungen kontrolliert.

In - Tab. 3 ist dargestellt, welche Indikatoren nach dieser Auswahl für die Analyse der drei Gesundheitsoutcomes übrig geblieben sind. Außerdem sind nun auch die jeweiligen Berechnungsgrundlagen auf Basis der Datendokumentation der INKAR-Datenbank [12] dargestellt. Der Blick auf die jeweils im Modell enthaltenen Merkmale macht deutlich, dass sich diese zwischen den drei Modellen unterscheiden. Wäre folglich nur eine Auswahl von regionalen Determinanten in allen Modellen berücksichtigt worden, hätte dies zu ineffizienten und/oder zu verzerrten Schätzwerten führen können. Zudem ist dieses Ergebnis - im Einklang mit Befunden zur Bedeutung von sozioökonomischen Determinanten auf der Individualebene [25] - dahingehend zu interpretieren, dass die sozioökonomischen Determinanten auch auf der Kontextebene für die betrachteten Gesundheitsindikatoren unterschiedlich relevant sind.

\section{Small-Area-Estimation (SAE)}

Bundesweit repräsentative Stichproben sind zumeist so bemessen, dass sie die
Tab. 4 Hierarchische Regressionsmodelle zu den Determinanten der Gesundheitsindikatoren auf Kreisebene. Datenbasis: GEDA 2009, INKAR 2009

\begin{tabular}{|c|c|c|c|c|c|c|}
\hline \multirow[b]{2}{*}{ Individualmerkmale } & \multicolumn{2}{|c|}{ Subjektive Gesundheit } & \multicolumn{2}{|c|}{ Rauchen } & \multicolumn{2}{|c|}{ Adipositas } \\
\hline & OR & [95\%-KI] & OR & [95\%-KI] & OR & [95\%-KI] \\
\hline \multicolumn{7}{|l|}{ Geschlecht } \\
\hline Männer & Ref. & & Ref. & & Ref. & \\
\hline Frauen & $1,20^{*}$ & $\begin{array}{l}{[1,12-} \\
1,28]\end{array}$ & $0,75^{*}$ & $\begin{array}{l}{[0,71-} \\
0,80]\end{array}$ & $0,87^{*}$ & $\begin{array}{l}{[0,80-} \\
0,94]\end{array}$ \\
\hline \multicolumn{7}{|l|}{ Alter } \\
\hline 18 bis 39 Jahre & Ref. & & Ref. & & Ref. & \\
\hline 40 bis 64 Jahre & $2,34^{*}$ & $\begin{array}{l}{[2,15-} \\
2,55]\end{array}$ & $0,70^{*}$ & $\begin{array}{l}{[0,66-} \\
0,75]\end{array}$ & $2,07^{*}$ & $\begin{array}{l}{[1,86-} \\
2,30]\end{array}$ \\
\hline 65 Jahr u. m. & $5,04^{*}$ & $\begin{array}{l}{[4,56-} \\
5,58]\end{array}$ & $0,17^{*}$ & $\begin{array}{l}{[0,16-} \\
0,20]\end{array}$ & $2,39^{*}$ & $\begin{array}{l}{[2,10-} \\
2,72]\end{array}$ \\
\hline Haushaltsgröße & $0,86^{*}$ & $\begin{array}{l}{[0,84-} \\
0,89]\end{array}$ & $0,87^{*}$ & $\begin{array}{l}{[0,85-} \\
0,89]\end{array}$ & $0,93^{*}$ & $\begin{array}{l}{[0,90-} \\
0,96]\end{array}$ \\
\hline \multicolumn{7}{|l|}{ Kontextmerkmale } \\
\hline \multicolumn{7}{|l|}{$\begin{array}{l}\text { Arbeitsmarkt und Ar- } \\
\text { beitslosigkeit }\end{array}$} \\
\hline $\begin{array}{l}\text { Arbeitslosenquote } \\
\text { Frauen }\end{array}$ & & & 0,89 & $\begin{array}{l}{[0,78-} \\
1,02]\end{array}$ & & \\
\hline $\begin{array}{l}\text { Arbeitslosenquote } \\
\text { Männer }\end{array}$ & & & $1,30^{*}$ & $\begin{array}{l}{[1,11-} \\
1,51]\end{array}$ & & \\
\hline $\begin{array}{l}\text { Einpersonen-Bedarfs- } \\
\text { gemeinschaften }\end{array}$ & & & $0,91^{*}$ & $\begin{array}{l}{[0,86-} \\
0,96]\end{array}$ & & \\
\hline $\begin{array}{l}\text { Erwerbsquote Män- } \\
\text { ner }\end{array}$ & & & 0,96 & $\begin{array}{l}{[0,92-} \\
1,00]\end{array}$ & & \\
\hline Sanktionsquote & 0,97 & $\begin{array}{l}{[0,93-} \\
1,00]\end{array}$ & $1,04^{*}$ & $\begin{array}{l}{[1,00-} \\
1,08]\end{array}$ & & \\
\hline SGB II - Quote & $1,07^{*}$ & $\begin{array}{l}{[1,01-} \\
1,13]\end{array}$ & & & & \\
\hline \multicolumn{7}{|l|}{$\begin{array}{l}\text { Wirtschaft und Reich- } \\
\text { tum }\end{array}$} \\
\hline $\begin{array}{l}\text { Bruttoinlandsprodukt } \\
\text { je Einwohner }\end{array}$ & 0,90 & $\begin{array}{l}{[0,80-} \\
1,00]\end{array}$ & 1,06 & $\begin{array}{l}{[0,98-} \\
1,14]\end{array}$ & 0,94 & $\begin{array}{l}{[0,88-} \\
1,01]\end{array}$ \\
\hline Dienstleistungsquote & 1,09 & $\begin{array}{l}{[0,97-} \\
1,22]\end{array}$ & $0,93^{*}$ & $\begin{array}{l}{[0,86-} \\
1,00]\end{array}$ & & \\
\hline Haushaltseinkommen & 0,96 & $\begin{array}{l}{[0,91-} \\
1,01]\end{array}$ & & & & \\
\hline Industriequote & $1,05^{*}$ & $\begin{array}{l}{[1,00-} \\
1,10]\end{array}$ & & & & \\
\hline \multicolumn{7}{|l|}{$\begin{array}{l}\text { Gesundheitliche Lage } \\
\text { und Versorgung }\end{array}$} \\
\hline Ambulante Pflege & $0,95^{*}$ & $\begin{array}{l}{[0,92-} \\
0,99]\end{array}$ & & & & \\
\hline Einwohner je Arzt & $1,10^{*}$ & $\begin{array}{l}{[1,03-} \\
1,17]\end{array}$ & & & $1,14^{*}$ & $\begin{array}{l}{[1,05-} \\
1,24]\end{array}$ \\
\hline Krankenhausbetten & 1,05 & $\begin{array}{l}{[0,99-} \\
1,10]\end{array}$ & & & $1,08^{*}$ & $\begin{array}{l}{[1,01-} \\
1,16]\end{array}$ \\
\hline $\begin{array}{l}\text { Lebenserwartung } \\
\text { Männer }\end{array}$ & & & 0,96 & $\begin{array}{l}{[0,92-} \\
1,01]\end{array}$ & $0,93^{*}$ & $\begin{array}{l}{[0,88-} \\
0,97]\end{array}$ \\
\hline Pflegebedürftige & $1,06^{*}$ & $\begin{array}{l}{[1,01-} \\
1,10]\end{array}$ & & & & \\
\hline Stationäre Pflege & & & & & $0,94^{*}$ & $\begin{array}{l}{[0,90-} \\
0,99]\end{array}$ \\
\hline
\end{tabular}


Tab. 4 Hierarchische Regressionsmodelle zu den Determinanten der Gesundheitsindikatoren auf Kreisebene. Datenbasis: GEDA 2009, INKAR 2009 (Fortsetzung)

$\begin{array}{lll}\text { Sonstige Faktoren } & & \\ \text { Baulandpreise } & 1,05 & {[0,99-} \\ & & 1,11]\end{array}$

Betreuungsquote

Kleinkinder

Einwohnerdichte

$0,94^{*}$

$[0,89$

$0,98]$

$1,05 \quad[0,99$

$1,11]$

Unfalltote

Exp(Konstante)

$0,19^{*}$

$[0,17-$

$0,21]$

1,07

$[0,97-$

$1,17]$

1,04

$0,11^{*}$

$[0,99-$

$1,09]$

Statistiken

Beobachtungen

Parameter

Log-Likelihood

$\mathrm{CHI}^{2}$ Modell

$\mathrm{P}\left(\mathrm{CHI}^{2}\right.$ Modell $\left.=0\right)$

MOR

$\mathrm{CH}{ }^{2} \mathrm{MOR}$

$\mathrm{P}\left(\mathrm{CH}^{2} \mathrm{MOR}=1\right)$

21009
15
-10905
1591,2
0,000
1,00
0,0

1,000

21028

14

$-12089$

1047,9

0,000

1,00

0,0

1,000

Alle einbezogenen Kontextmerkmale wurden z-standardisiert. Die zugehörigen Odds Ratios spiegeln jeweils den Einfluss der Veränderung des jeweiligen Indikators um eine Standardabweichung im Vergleich zum Mit-

telwert wider. MOR = Median Odds Ratio (Maß für den Zusammenhang zwischen Kontext und Outcome nach Kontrolle für die enthaltenen erklärenden Varianten, 1,00: kein Zusammenhang). ${ }^{*}$-Wert: $p<0.05$

wichtigsten Zielgrößen für das gesamte Erhebungsgebiet mit hinreichender Genauigkeit schätzen können. Sie reichen allerdings nicht aus, um diese Zielgrößen auch direkt für kleinräumige Gebiete wie Kreise zu ermitteln. Mit den Verfahren zur Small-Area-Estimation (SAE) stehen statistische Ansätze zur Verfügung, die diese Schätzung indirekt unter der $\mathrm{Zu}$ hilfenahme amtlicher Statistiken aus anderen Quellen ermöglichen [4].

Prinzipiell basiert die Small-Area-Estimation auf den Regressionsmodellen, die auch in der Epidemiologie zum Einsatz kommen. Bei der SAE wechselt allerdings das Erkenntnisinteresse bei der statistischen Inferenz: So soll nicht von einer Stichprobe aus Individuen auf eine Grundgesamtheit von Individuen geschlossen werden, sondern auf Basis von Regionalindikatoren und einer Stichprobe von Individuen auf die Eigenschaften einer Grundgesamtheit aus Regionen. Im Zuge der SAE werden dazu regionalisierbare Individualdaten mit anderen regionalisierbaren Datenkörpern verknüpft (üblicherweise amtliche Statistiken). Auf Basis dieser zusammengeführten Datensätze kann anschließend eine regressions- basierte Schätzung der interessierenden Parameter (Prävalenzen, Inzidenzen, Inanspruchnahmeraten) in den „Small Areas" vorgenommen werden. Der Ansatz der SAE wurde in den letzten Jahren bereits mehrfach für kleinräumige Schätzungen von epidemiologischen Parametern genutzt $[26,27,28,29,30,31]$. In den USA werden die Verfahren in der Regel auf der Ebene der sogenannten counties angewendet, die durchschnittlich eine kleinere Bevölkerung aber eine größere Fläche als Kreise und kreisfreien Städte in Deutschland haben. Generell ist bei der Interpretation von Aggregatdaten zu beachten, dass sich die Wahl der Ebene der Regionalisierung von aggregierten Daten auf die Ergebnisse der Analysen auswirkt (sogenanntes Modifiable-Area-Unit-Problem (MAUP) [32]).

Bei der Small-Area-Estimation wird die Prävalenz in den kleinräumigen Gebieten nicht direkt aus der Stichprobe, sondern indirekt aus dem Zusammenhang zwischen der - über Regionalindikatoren abgebildeten - Sozialstruktur der kleinräumigen Gebiete und der Prävalenz des Outcomes auf der Individualebene ermittelt. Als Regionalindikato- ren sollten dabei Merkmale gewählt werden, von denen angenommen werden kann, dass sie auch auf der Individualebene mit dem abhängigen Merkmal assoziiert sind. Je mehr relevante Indikatoren in das Modell aufgenommen werden, desto aussagekräftiger sind dabei die Vorhersageergebnisse der Small-Area-Estimation. Der Zusammenhang zwischen Stichprobe und externen Daten wird bei der Small-Area-Estimation über ein Regressionsmodell für hierarchische Daten (sogenanntes Multi-level- beziehungsweise Mehrebenenmodell beziehungsweise auch Zufalls-Effekt-Modell) hergestellt $[33,34]$. In Mehrebenenmodellen können sowohl die Konstante des Regressionsmodells, als auch die Effekte individueller Merkmale als kontextabhängig modelliert werden (Random-Intercept- beziehungsweise Random-Effect/RandomSlope-Modelle). Die Grundgleichung linearer Mehrebenen-Regressionsmodelle ist in Formel 1 dargestellt.

\section{Formel 1}

\section{Grundgleichung der Mehrebenenanalyse mit einer erklärenden Variable}

$Y_{i, k}=b_{0, k}+b_{1, k} X_{1, i}+e_{i, k}$

$\mathrm{Y}_{\mathrm{i}, \mathrm{k}}$ : Abhängiges Merkmal das zwischen Individuen $(i)$ und Kontexten $(k)$ variiert

$\mathrm{b}_{\mathbf{o}, \mathrm{k}}$ : Konstante, die zwischen Kontexten variiert. Sie lässt sich in einen fixen und einen variablen, kontextspezifischen Anteil zerlegen: $\mathrm{b}_{\mathrm{o}, \mathrm{k}}=b_{\mathrm{o}}+\mathrm{u}_{\mathrm{o}, \mathrm{k}}$

$\mathbf{b}_{\mathbf{1}, \mathbf{k}}$ : Effekt der erklärenden Variable $\mathrm{X}_{\mathbf{1}}$, der zwischen den Kontexten variiert, er lässt sich in einen fixen und einen variablen, kontextspezifischen Anteil zerlegen: $b_{1, k}=b_{1}+u_{1, k}$

$\mathbf{e}_{\mathbf{i}, \mathbf{k}}$ : Residuum des Individuums ( $i$ )

In dieser Studie wird eine Abwandlung des geschilderten idealtypischen Ansatzes der SAE vorgenommen. Dabei werden in den Modellen nicht nur Merkmale auf der Kontextebene herangezogen, sondern 
auch Individualmerkmale. Dieses Vorgehen wurde schon mehrfach bei der kleinräumigen Schätzung von Gesundheitsoutcomes auf Basis von Surveydaten und amtlichen Statistiken erfolgreich umgesetzt und soll zu verbesserten Schätzwerten führen [30, 31]. Es werden hierarchisch logistische Regressionsmodelle verwendet, um Regressionsparameter für individuelle und kontextbezogene Merkmale zu ermitteln. Die Schätzung baut auf diesen Parametern auf, allerdings werden die Ausprägungen der individuellen Prädiktoren durch deren Mittelwert im jeweiligen Kontext eingesetzt. Diese Mittelwerte werden dabei in der Regel nicht auf Basis der Stichprobe generiert, sondern aus externen Quellen bezogen. Nachfolgend wird in den Analysen für das Alter und das Geschlecht der Befragten sowie für ihre Haushaltsgröße kontrolliert. Für die kleinräumige Schätzung werden dann aber die regionalen Anteile der verwendeten Altersgruppen, die regionale Frauenquote sowie die mittlere HaushaltsgröBe in den Regionen aus der INKAR-Datenbank bezogen und mit den Parametern zusammengeführt. Im Resultat erhöht sich bei dieser Vorgehensweise die statistische Güte der Vorhersage, da die auf Basis von Individualdaten geschätzten Modellparameter mit einer höheren statistischen Genauigkeit berechnet werden können als die Kontexteffekte. Zufällige Effekte werden in die Schätzung nicht miteinbezogen. Der auf der Schätzung zufälliger Effekte aufbauende BLUP-Schätzer (Best Linear Unbiased Predictor) wird nicht verwendet, da die Fallzahlen auf der Kreisebene als nicht ausreichend angesehen wurden, um stabile BLUP-Schätzwerte zu erlangen. Die resultierende Gleichung bei der Schätzung für das verwendete logistische Mehrebenenmodell ist in Formel 2 dargestellt.

\section{Formel 2}

\section{Kleinräumige Vorhersage auf Basis von Individual- und Kontextmerkmalen mit logistischen Mehrebenenmodellen}

$$
\text { Logit } \hat{Y}_{k}=\hat{b}_{0}+\sum \hat{b}_{z} Z_{k}+\sum \hat{b}_{x} \bar{X}_{k}
$$

\section{Analysestrategie}

In der Analyse werden zuerst logistische Mehrebenenmodelle für die drei Gesundheitsoutcomes berechnet. Dabei gehen sowohl Individualmerkmale als auch Kontextindikatoren ein. Aufbauend auf den Analysen wird eine Small-Area-Estimation durchgeführt und grafisch anhand von thematischen Karten dargestellt. Das Ergebnis der SAE wird anschließend exemplarisch für die Prävalenz von Adipositas anhand von kleinräumigen Prävalenzen aus dem Mikrozensus 2005 des Statistischen Bundesamtes überprüft. Ein Vergleich war nur für diesen Indikator möglich, da die subjektive Gesundheit im Mikrozensus nicht erhoben wird und sich die Strukturen des Tabakkonsums in Deutschland seit 2005 so rapide verändert haben [35], dass ein Vergleich zwischen 2005 und 2009 nicht möglich ist.

Der Mikrozensus ist eine jährliche, einprozentige Stichprobe der Haushalte in Deutschland, die von den Statistischen Ämtern der Länder erhoben wird [36]. Aufgrund seines umfassenden Stichprobenumfanges eröffnet allein der Mikrozensus für Deutschland die Möglichkeit, auf Basis der herkömmlichen Methode der statistischen Inferenz für kleinräumige Gebiete Mittelwerte und Prävalenzen mit hoher statistischer Genauigkeit zu schätzen. In der Zusatzbefragung zur Gesundheit, die in mehrjährigen Abständen stattfindet, werden auch einige wenige Gesundheitsindikatoren erhoben. Diese Daten sollen nachfolgend genutzt werden, um exemplarisch am Beispiel der Prävalenz von Adipositas die Ergebnisse der SAE zu überprüfen. Die Regionalisierung der Daten des Mikrozensus ist zur Gewährleistung des Datenschutzes nur unter strengen Auflagen möglich. Das Robert Koch-Institut hat daher das Statistische Bundesamt mit einer Sonderauswertung des Mikrozensus beauftragt und kleinräumige Prävalenzen für Adipositas auf der Ebene der 97 Raumordnungsregionen im Jahr 2005 erhalten [37]. Obwohl der Bezugszeitraum von der GEDAStudie 2009 abweicht, sollten sich auf Basis dieser Daten insgesamt vergleichbare regionale Disparitäten ergeben. Die Prävalenz von Adipositas ist zwischen den
Mikrozensus-Erhebungen 2005 und 2009 von $13,6 \%$ auf $14,7 \%$ gestiegen $[38,39]$. Es steht daher zu erwarten, dass die aus der GEDA-Studie 2009 ermittelten Prävalenzen über denen des Mikrozensus 2005 liegen. Für den Vergleich der Ergebnisse mussten die anhand der SAE ermittelten Prävalenzen für die Kreisebene, gewichtet nach der Bevölkerungsgröße der Kreise, für die 97 Raumordnungsregionen in Deutschland aggregiert werden.

Alle Berechnungen werden mit dem Programm Stata 11.1 SE für die Bevölkerung im Alter ab 18 Jahren durchgeführt [40]. Die Mehrebenenmodelle mussten dabei ungewichtet berechnet werden, da die in Stata verfügbaren Prozeduren noch keine Berücksichtigung von Gewichtungsfaktoren erlauben. Die anhand der SAE vorhergesagten Prävalenzen für die Kreise mussten daher, um Inkonsistenzen in der Darstellung und Probleme bei der Interpretation der Ergebnisse zu vermeiden, nachträglich für die gewichtungsbedingten Abweichungen korrigiert werden. Durch den Korrekturfaktor wurde der bevölkerungsgewichtete Mittelwert der Prävalenzen aus der SAE auf Kreisebene auf die gewichtete Prävalenz aus den Individualdaten zentriert. Nach der Korrektur entspricht der gewichtete Mittelwert der vorhergesagten Prävalenzen aus der SAE der für die GEDA-Studie 2009 berichteten Prävalenz für das Bundesgebiet. Die deskriptiven Darstellungen auf Basis der Individualdaten der GEDA-Studie 2009 wurden mit den bereitgestellten Hochrechnungsfaktoren gewichtet. Die thematischen Karten wurden mit dem Programm RegioGraph Analyse 11 von GfK Geomarketing erstellt und anschließend anhand eines digitalen Bildbearbeitungsprogramms überarbeitet. Die Einfärbung der thematischen Karten wurde mit dem Algorithmus „Statistisch verteilt" (5 Gruppen) auf Basis der Annahme einer Normalverteilung der Prävalenzen über die Kreise durchgeführt. Die Grenzen der untersten und obersten Kategorie werden dabei jeweils zwei Standardabweichungen vom Mittelwert entfernt gesetzt, die übrigen drei Kategorien werden gleichmäßig dazwischen verteilt. Trifft die Annahme der Normalverteilung zu, ist die Anzahl von Flächen im Bereich um den Mittelwert der Prävalen- 


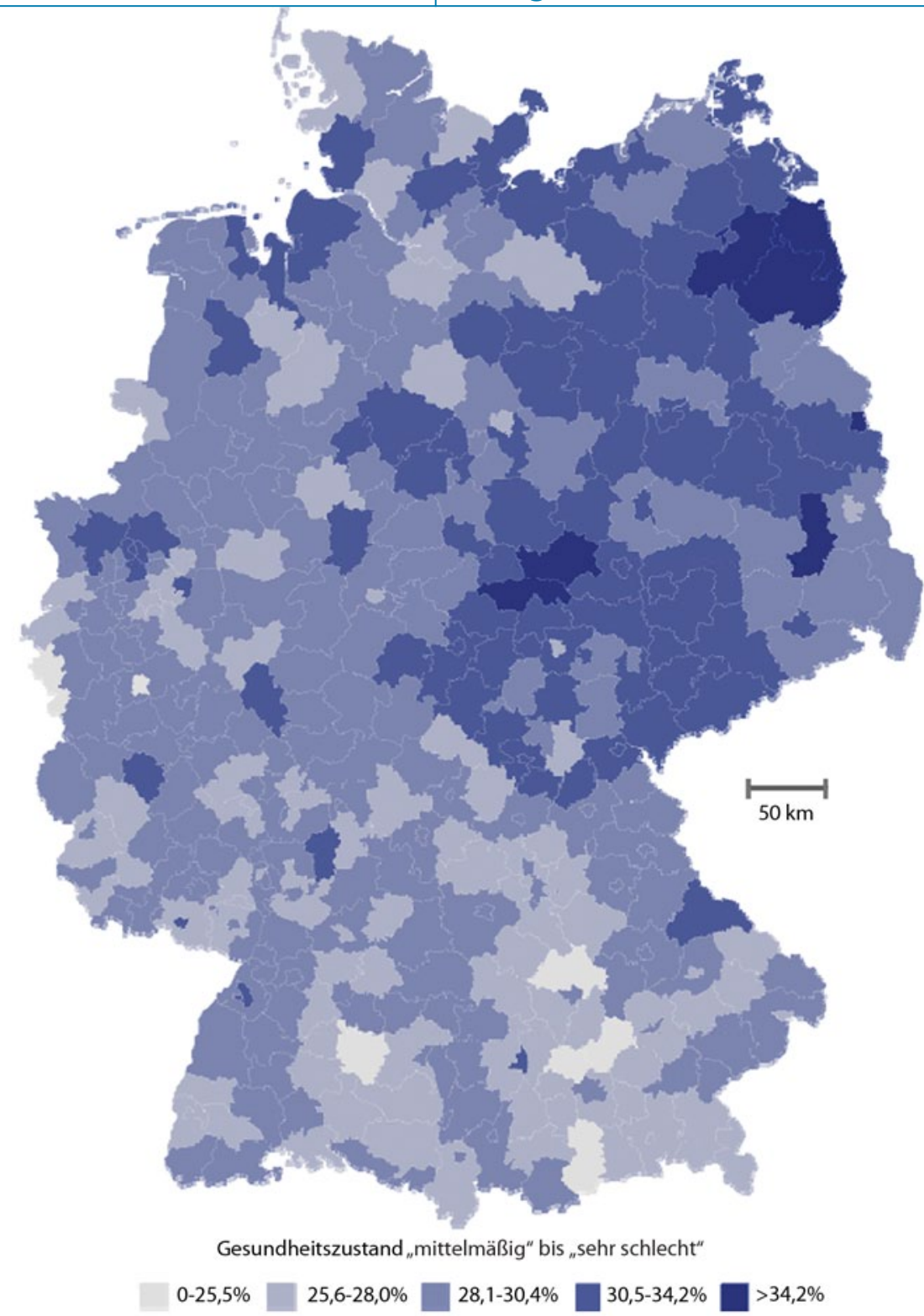

Abb. $1 \Delta$ a Anteil allgemeiner Gesundheitszustand "mittelmäßig" bis sehr schlecht auf Kreisebene in Prozent. Datenbasis: GEDA 2009 (Alter 18+ Jahre), INKAR 2009. b Rauchquote auf Kreisebene in Prozent. Datenbasis: GEDA 2009 (Alter 18+ Jahre), INKAR 2009. c Prävalenz von Adipositas auf Kreisebene in Prozent. Datenbasis: GEDA 2009 (Alter 18+ Jahre), INKAR 2009

zen am größten und nimmt mit zunehmendem Abstand zu ihm ab.

\section{Ergebnisse}

In $\bullet$ Tab. 4 sind die Ergebnisse der logistischen Mehrebenenmodelle dargestellt. Ausgewiesen werden die Effekte jeweils als Odds Ratios (OR) mit dem zugehörigen 95\%-Konfidenzintervall. Um die Interpretation der Kontexteffekte zu erleichtern, wurden diese Variablen z-transformiert. Die Odds Ratios der Kontextmerkmale beschreiben also den Effekt, den die
2007. Im Vergleich der Effekte der Individual- und der Kontextmerkmale zeigt sich, dass die Einflussstärke der Kontextmerkmale deutlich unter der Bedeutung der Individualmerkmale liegt. Die Güte der drei Regressionsmodelle im Hinblick auf die Möglichkeiten zur Small-Area-Estimation lässt sich anhand des Maßes Median Odds Ratio (MOR) vergleichen. Dieses Maß beschreibt analog zum Intraklassenkorrelationskoeffizienten bei der hierarchischen linearen Regression, wie sehr das Risiko des jeweiligen Outcomes zwischen den Kreisen variiert, nachdem für die im Modell enthaltenen Kontextmerkmale kontrolliert wurde. Je stärker der Wert des MOR von 1,0 abweicht, desto mehr durch das Modell unberücksichtigte Heterogenität besteht zwischen den Kreisen im Hinblick auf das Outcome. Für die Modelle zur subjektiven Gesundheit und zum Rauchverhalten deuten die Werte an, dass keine unberücksichtigte Heterogenität zwischen den Kreisen vorhanden ist. Dies wird durch den zugehörigen $\chi^{2}$-Test bestätigt, der überprüft, ob es nach Kontrolle der erklärenden Variablen noch eine signifikante Variation des Outcomes über die Kontexte gibt. Demgegenüber zeigt sich beim Risiko von Adipositas, dass das Modell nicht optimal ist $(\mathrm{MOR}=1,2)$. Der zugehörige $\mathrm{p}$-Wert des $\chi^{2}$ Tests $(\mathrm{p}<0.05)$ deutet hier darauf hin, dass die fortbestehenden Differenzen zwischen den Kreisen statistisch bedeutsam sind. Aufbauend auf den hierarchischen Modellen werden nachfolgend die Prävalenzen der drei Gesundheitsindikatoren auf Kreisebene bestimmt und als thematische Karten dargestellt.

In den $\bullet$ Abb. $1 a, b, c$ werden die mittels der Small-Area-Estimation für die Bevölkerung im Alter ab 18 Jahre vorhergesagten Prävalenzen der drei Outcomes auf Kreisebene dargestellt. Bemerkenswert ist dabei zuvorderst, dass bei allen drei Merkmalen beträchtliche regionale Disparitäten bestehen und dass sich diese regionalen Verteilungsmuster zwischen den Outcomes deutlich unterscheiden. Insgesamt schätzen 29,6\% der Befragten in der GEDA-Studie 2009 ihre Gesundheit nicht „mittelmäßig“ bis „sehr schlecht“ ein. Die vorhergesagte Prävalenz in den Kreisen variiert aber zwischen $15,9 \%$ und $44,6 \%$ (Interquartilsabstand IQR $=6,9 \%$; Varia- 
tionskoeffizient $\mathrm{CV}=16,3 \%)$. Es zeigt sich dabei ein Gefälle zwischen hohen Prävalenzen im Nordosten und geringen Prävalenzen im Süden der Republik. Auch beim Rauchverhalten zeigen sich beträchtliche Differenzen. Die Rauchquote in Deutschland betrug im Jahr 2009 laut den Daten der GEDA-Studie $29,9 \%$. Sie variierte dabei allerdings zwischen $14,9 \%$ und $44,5 \%$ zwischen den Kreisen (IQR =6,1\%, $\mathrm{CV}=15,6 \%)$. Das geografische Muster der Verteilung der Rauchquote ist dabei nicht so eindeutig. Nach den Daten der GEDAStudie 2009 liegt der Anteil von Adipositas in Deutschland bei 16,0\% für die Bevölkerung im Alter ab 18 Jahren. Die Ergebnisse der SAE deuten darauf hin, dass auch diese Prävalenz in beträchtlichem Maße zwischen den Kreisen variiert. So liegt die Prävalenz in einigen Kreisen bei nur $10 \%$, während sie in den Kreisen mit der höchsten Prävalenz über 20\% beträgt $(\mathrm{IQR}=3,5 \% ; \mathrm{CV}=16,3 \%)$. Es zeigt sich dabei ein Gefälle hinsichtlich der Prävalenz von Nordost nach Südwest. Zusammenfassend machen die als thematische Karten dargestellten Ergebnisse der Small-Area-Estimation deutlich, dass es in Deutschland beträchtliche regionale Disparitäten hinsichtlich der betrachteten Gesundheitsindikatoren gibt.

In - Abb. 2 wird abschließend die Prävalenz von Adipositas aus der SmallArea-Estimation auf Basis von GEDA 2009 mit der zugehörigen Prävalenz aus dem Mikrozensus 2005 verglichen. Für den Vergleich beider Prävalenzen mussten die Ergebnisse der SAE, die für die Kreisebene vorlagen, für die 97 Raumordnungsregionen in Deutschland aggregiert werden. Erwartungsgemäß liegen die Prävalenzen auf Basis des Mikrozensus 2005 im Durchschnitt unter denen, die anhand der GEDA-Studie 2009 ermittelt wurden. Die Korrelation beider Prävalenzen beträgt $\mathrm{r}=0.70$. Aus der Abbildung geht hervor, dass eine relativ große Übereinstimmung zwischen den beiden Prävalenzen besteht. Die Güte der Vorhersage auf Basis der GEDA-Daten nimmt dabei auch für Regionen mit besonders hohen oder niedrigen Prävalenzen nicht ab. Allerdings gibt es eine systematische Differenz zwischen beiden Datenquellen. Die auf Basis von GEDA ermittelten Werte liegen, gewichtet nach der Bevölkerung der

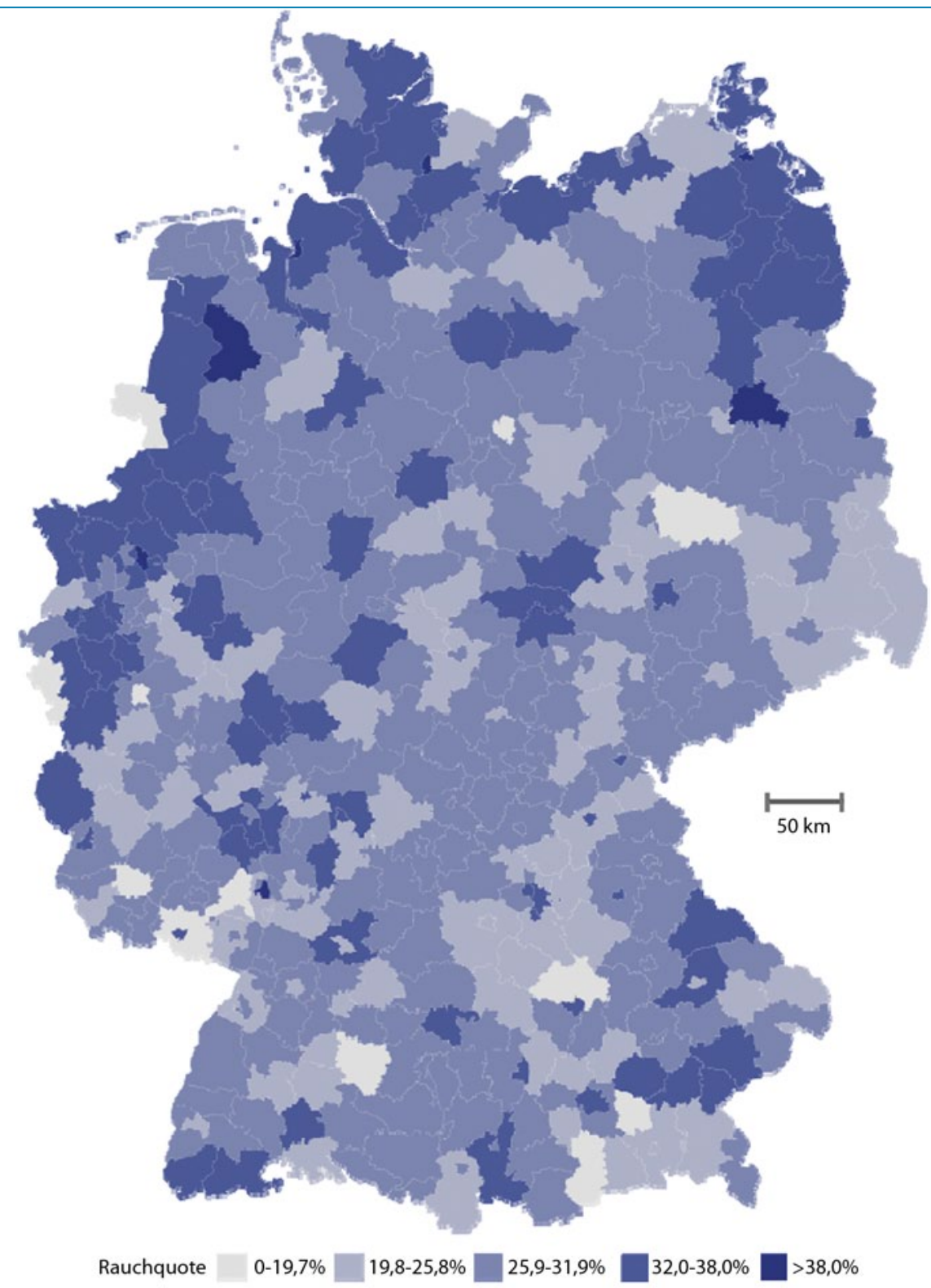

Abb. $1 \Delta$ Fortsetzung (b)

Regionen, im Durchschnitt 2,5\% über den Werten des Mikrozensus 2005. Diese Differenz erscheint aber im Hinblick auf die methodischen Differenzen beider Studien (PAPI versus CATI) und ihren zeitlichen Abstand (2005 versus 2009) als relativ gering und die vorgefundene Korrelation der Prävalenzen als relativ gut.

\section{Fazit}

Im vorliegenden Beitrag wurde ein Verfahren zur Gewinnung kleinräumiger Schätzwerte für Gesundheitsindikatoren durch die Kombination von amtlichen Strukturdaten und repräsentativen Mi- krodaten aus dem Gesundheitsmonitoring am Robert Koch-Institut vorgestellt und angewendet. Es liegen auf Basis der gewählten Methode Ergebnisse für den allgemeinen Gesundheitszustand sowie für die Prävalenzen des aktuellen Rauchens und von Adipositas vor. Die Ergebnisse sprechen dafür, dass in Deutschland hinsichtlich der drei Gesundheitsindikatoren beachtliche regionale Disparitäten bestehen. Für die Prävalenz von Adipositas wurde darüber hinaus ein Vergleich mit kleinräumigen Ergebnissen aus dem amtlichen Mikrozensus 2005 auf der Ebene der Raumordnungsregionen vorgenommen. Der Mikrozensus ist 


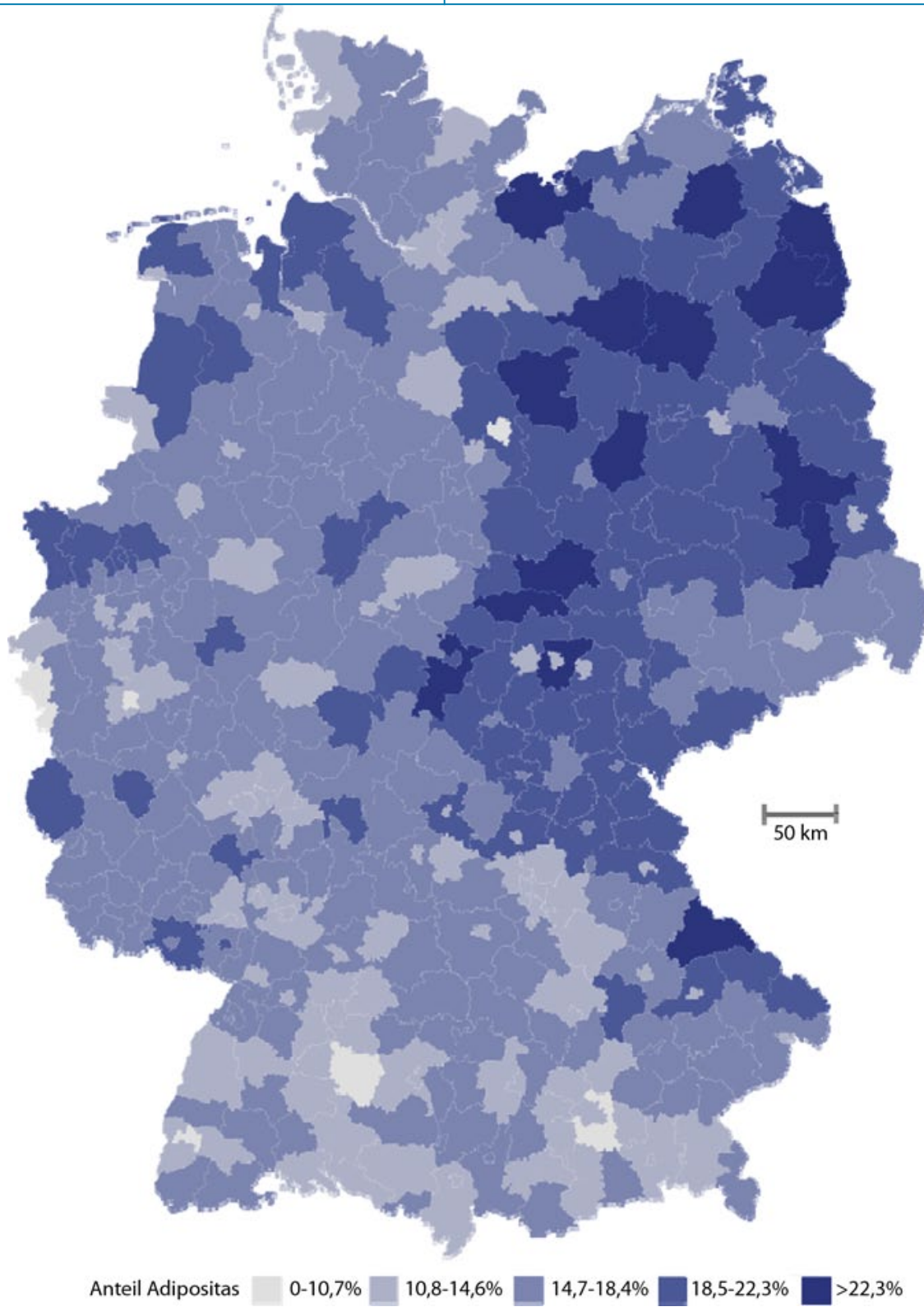

Abb. $1 \Delta$ Fortsetzung $(\mathbf{c})$

die einzige Datenquelle in Deutschland, die aufgrund ihrer umfangreichen Stichprobe eine direkte Berechnung regionaler Prävalenzen erlaubt. Es wurde eine große Übereinstimmung beider Datenquellen festgestellt, sodass anzunehmen ist, dass die generierten Prävalenzen insgesamt ein vergleichsweise realistisches Bild der regionalen Disparitäten hinsichtlich der Prävalenz von Adipositas in Deutschland zeichnen. Einschränkend ist hierzu anzumerken, dass bei diesem Vergleich ein Modifiable-Area-Unit (MAUP)Problem besteht, da er lediglich auf der (höheren) Ebene der Raumordnungsregionen durchgeführt werden konnte. Al- ternative Zusammenfassungen von Kreisen könnten zu unterschiedlichen Korrelationen und Schlussfolgerungen führen. Der gewählte Ansatz der Small-Area-Estimation, der auf hierarchischen Regressionsmodellen basiert, wurde bereits mehrfach erfolgreich angewendet [28, $30,31,41]$. In den USA wurden auf Basis der Daten des Behavioral Risk Factor Surveillance Systems (BRFSS) der Centers of Disease Control and Prevention (CDC) Distrikte mit einer besonderen Priorität für die Prävention des Rauchens und von Adipositas identifiziert [30, 31]. In England wurden entsprechende Verfahren genutzt, um nach strukturellen De- terminanten zur Förderung der sportlichen Aktivität zu suchen. Arbeiten, welche die regionale Verteilung der Krankheitslast bei chronisch-degenerativen Erkrankungen in den USA nachzeichnen, deuten zudem darauf hin, dass die SAE auch für die medizinische Bedarfsplanung von Bedeutung sein kann [28]. Für Deutschland ist bisher keine Anwendung des Verfahrens für die verwendeten Gesundheitsindikatoren dokumentiert, allerdings wird das Verfahren auch hier zunehmend diskutiert [5]. Angesichts des international vielfach wahrgenommenen Bedeutungszuwachses der "Spatial Epidemiology" [41] steht aber zu erwarten, dass in Zukunft mehr Analysen zu diesem Thema publiziert werden.

Limitationen des Vorgehens ergeben sich durch die Auswahl der kontextuellen Determinanten und die begrenzte Stichprobengröße der GEDA-Studie 2009. Bei der Auswahl von Kontextmerkmalen gibt es einerseits die Möglichkeit einer streng theoriegeleiteten Auswahl und andererseits einer statistischen Auswahl der Indikatoren über $\mathrm{p}$-Werte. Die Autoren haben sich für eine statistische Auswahl aus einer inhaltlich begründeten Vorauswahl von relevanten und verfügbaren Merkmalen entschieden. Dabei wurde ein möglichst schwaches Zurückweisungskriterium verwendet, damit relevante, aber nicht statistisch bedeutsame Merkmale nicht vorschnell ausgeschlossen werden. Dieses Vorgehen ist einerseits dem vergleichsweise geringen Wissensstand zur Relevanz der in der INKAR Datenbank 2009 verfügbaren Indikatoren für die gesundheitliche Lage der Bevölkerung in Deutschland (für andere Staaten wie England oder die USA liegen mehr Erkenntnisse vor [17]) und andererseits dem statistisch aufwendigen Verfahren der Mehrebenenanalyse geschuldet. Zudem ermöglichte es aber auch aufzuzeigen, dass die verschiedenen Kontextindikatoren für die drei betrachteten Outcomes von unterschiedlicher Relevanz sind. Die aus der begrenzten Stichprobengröße von repräsentativen Studien resultierenden inferenzstatistischen Probleme waren der Ausgangspunkt für die Wahl und Anwendung der Small-Area-Estimation. Die Mehrebenenmodelle ermöglichen, trotz der - für den 
Zweck der kleinräumigen Analyse - geringen Fallzahl der GEDA-Studie 2009 zu hinreichend genauen Ergebnissen zu kommen. Bei der kleinräumigen Vorhersage der Prävalenzen wurde allerdings darauf verzichtet, die zufälligen Abweichungen (Random Intercept) zu berücksichtigen, da die Fallzahl den Autoren für ein entsprechendes Vorgehen zu gering erschien. Es besteht aber für zukünftige Analysen die Möglichkeit, mehrere Wellen der GEDA-Studie zusammenzuspielen und so eine ausreichende Fallzahl für vertiefende Analysen zu generieren. Dies ist insbesondere für Outcomes von Bedeutung, bei denen es nach Berücksichtigung kontextueller Determinanten noch eine signifikante Variation zwischen den Kontexten gibt (abzulesen am MOR beziehungsweise dem p-Wert der Varianz der zufälligen Effekte). Es ist geplant, die vorliegenden Analysen auf Basis eines solchen Datensatzes zu replizieren und auszubauen.

Zusammengenommen stellen die vorgestellten Ergebnisse einen ersten Schritt dar, Gesundheitsindikatoren auf Basis bevölkerungsrepräsentativer Daten zu regionalisieren. Die Ergebnisse der GEDA-Studie 2009 erscheinen in dieser Hinsicht vielversprechend und sollten den Ausgangspunkt für weitere Forschungen auf diesem Gebiet bilden. Die Daten des Gesundheitsmonitorings am Robert Koch-Institut liefern hierfür eine wichtige Grundlage. Sie stellen für eine Vielzahl von Gesundheitsindikatoren bei Kindern und Jugendlichen sowie bei Erwachsenen bundesweit repräsentative Befragungs- und Untersuchungsdaten bereit, die anhand der vorgestellten Methode der Small-Area-Estimation regionalisiert werden können. Die Erhebungen des Gesundheitsmonitorings werden regelmäßig durchgeführt und erlauben somit perspektivisch auch Aussagen darüber, wie sich die regionalen Disparitäten in der gesundheitlichen Lage der Bevölkerung Deutschlands entwickeln.

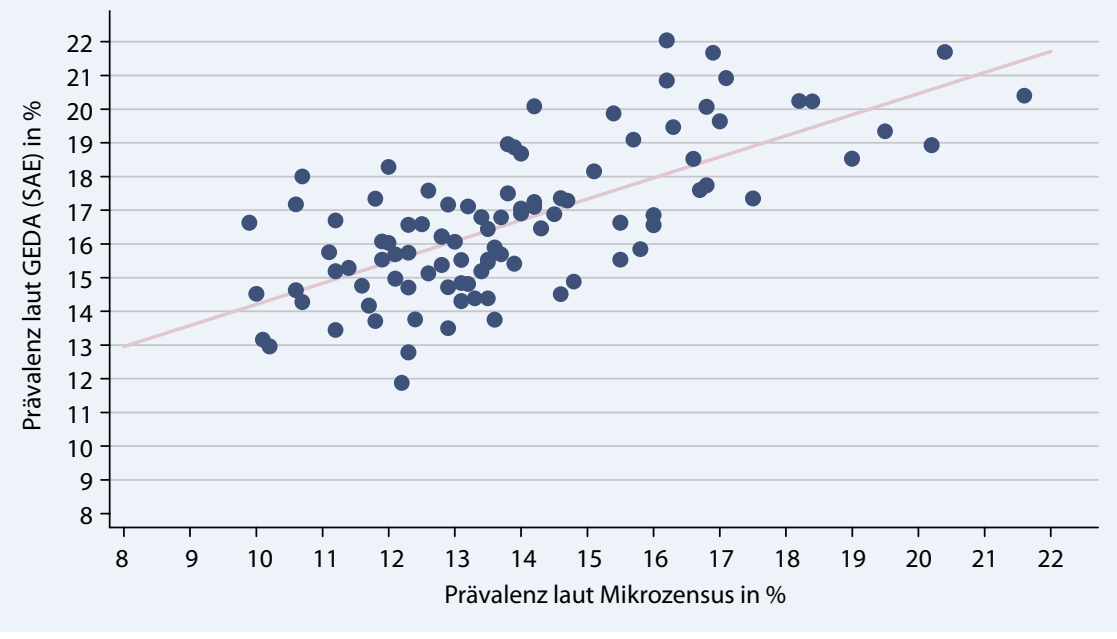

Abb. $2 \Delta$ Vergleich der Prävalenz von Adipositas auf Ebene der Raumordnungsregionen zwischen den Studien GEDA 2009 (Ergebnis der Small Area Estimation) und dem Mikrozensus 2005 (direkte Schätzung). Datenbasis: GEDA 2009, Mikrozensus 2005

\section{Korrespondenzadresse}

\section{Dr. L.E. Kroll}

FG 27, Abteilung für Epidemiologie und Gesundheitsberichterstattung, Robert Koch-Institut

13302 Berlin

L.Kroll@rki.de

Interessenkonflikt. Der korrespondierende Autor gibt an, dass kein Interessenkonflikt besteht.

\section{Literatur}

1. Kurth B-M, Lange C, Kamtsiuris $\mathrm{P}$, Hölling H (2009) Gesundheitsmonitoring am Robert Koch-Institut. Sachstand und Perspektiven. Bundesgesundheitsblatt Gesundheitsforschung Gesundheitsschutz 52:557-570

2. Gaber E, Wildner M (2011) Sterblichkeit, Todesursachen und regionale Unterschiede. Robert Koch-Institut, Berlin

3. Zentralinstitut für die kassenärztliche Versorgung Z (2011) versorgungsatlas.de. In: ZI, Berlin

4. Rao JNK (2003) Small area estimation. John Wiley, Hoboken

5. Voigt M (2009) Small Area Estimation: Die Schätzer von Fay-Herriot und Battese-Harter-Fuller. Wirtsch Stat 2:179-183

6. RKI (2011) Daten und Fakten: Ergebnisse der Studie "Gesundheit in Deutschland aktuell 2009«. In: Beiträge zur Gesundheitsberichterstattung des Bundes. Robert Koch-Institut, Berlin

7. Gabler S, Häder S (1999) Generierung von Telefonstichproben mit TelSuSa. ZUMA-Nachrichten 44:138-143

8. Gabler S, Häder S (Hrsg) (2002) Telefonstichproben - Methodische Innovationen und Anwendungen in Deutschland. Waxmann, München

9. Müters S, Lippe E von der, Kamtsiuris P et al (2010) Dokumentation zur Response in der Studie Gesundheit in Deutschland aktuell 2009. In: RKI (Hrsg) Methodische Beiträge zur Studie Gesundheit in Deutschland aktuell. RKI, Berlin, S 1-14
10. Idler EL, Benyamini Y (1997) Self-rated health and mortality: a review of twenty-seven community studies. J Health Soc Behav 38:21-37

11. Mensink G, Lampert T, Bergmann E (2005) Übergewicht und Adipositas in Deutschland 1984-2003. Bundesgesundheitsbl Gesundheitsforsch Gesundheitsschutz 48:1348-1356

12. BBSR (2009) INKAR 2009 - Indikatoren, Karten und Graphiken zur Raum- und Stadtentwicklung in Deutschland und in Europa. Bundesinstitut für Bau-, Stadt- und Raumforschung im Bundesamt für Bauwesen und Raumordnung, Wiesbaden

13. Starfield B (2007) Pathways of influence on equity in health. Soc Sci Med 64:1355

14. Wilkinson RG (2005) The impact of inequality: how to make sick societies healthier. New Press: Distributed by W.W. Norton, New York

15. Mackenbach JP (2006) Health Inequalities: Europe in Profile. UK Presidency of the EU, Rotterdam

16. Mielck A (2005) Soziale Ungleichheit und Gesundheit: Empirische Ergebnisse, Erklärungsansätze, Interventionsmöglichkeiten. Hans Huber, Bern

17. Macintyre S, Ellaway A, Cummins S (2002) Place effects on health: how can we conceptualise, operationalise and measure them? Soc Sci Med 55:125139

18. Kroll LE (2010) Sozialer Wandel, soziale Ungleichheit und Gesundheit. Die Entwicklung sozialer und gesundheitlicher Ungleichheiten in Deutschland zwischen 1984 und 2006. VS, Wiesbaden

19. Lampert T, Kroll LE (2010) Armut und Gesundheit. GBE kompakt 5:1-9

20. Lampert T, Saß A-C, Häfelinger M, Ziese T (2005) Armut, soziale Ungleichheit und Gesundheit. Expertise des Robert Koch-Instituts zum 2. Armutsund Reichtumsbericht der Bundesregierung. Robert Koch-Institut, Berlin

21. Janßen C, Grosse FK, Ommen O (2009) Sozial Ungleichheit und gesundheitsbezogene Versorgung. In: Richter M, Hurrelmann K (Hrsg) Gesundheitliche Ungleichheit. Grundlagen, Probleme, Konzepte. VS-Verlag, Wiesbaden, S 141-155

22. Lampert T, Kroll LE, Dunkelberg A (2007) Soziale Ungleichheit der Lebenserwartung in Deutschland. Polit Zeitgesch 42:11-18

23. Rabe-Hesketh S, Skrondal A (2008) Multilevel and longitudinal modeling using stata. Stata Press, College Station, TX 
24. Judd CM, McClelland GH, Ryan CS (2009) Data analysis: a model comparison approach. Routledge, New York

25. Lampert T, Kroll LE (2009) Messung des sozioökonomischen Status in sozialepidemiologischen Studien. In: Richter M, Hurrelmann K (Hrsg) Gesundheitliche Ungleichheit. Grundlagen, Probleme, Konzepte. VS-Verlag, Wiesbaden, S 309-3334

26. Congdon P, Shouls S, Curtis S (1997) A multi-level perspective on small-area health and mortality: a case study of England and Wales. Int J Popul Geogr 3:243-263

27. Congdon P (2010) A multilevel model for comorbid outcomes: obesity and diabetes in the US. Int J Environ Res Public Health 7:333-352

28. Congdon P (2009) A multilevel model for cardiovascular disease prevalence in the US and its application to micro area prevalence estimates. Int J Health Geogr 8:6

29. Cochrane T, Davey RC, Gidlow C et al (2009) Small area and individual level predictors of physical activity in urban communities: a multi-level study in Stoke on Trent, England. Int J Environ Res Public Health 6:654-677

30. Li W, Land T, Zhang Z et al (2009) Small-area estimation and prioritizing communities for tobacco control efforts in Massachusetts. Am J Public Health 99:470-479

31. Li W, Kelsey JL, Zhang Z et al (2009) Small-area estimation and prioritizing communities for obesity control in Massachusetts. Am J Public Health 99:511-519

32. Openshaw S (1984) Ecological fallacies and the analysis of areal census data. Environ Plan A 16:17-31

33. Leeuw J de, Meijer E (2008) Handbook of multilevel analysis. Springer, New York

34. Langer W (2008) Mehrebenenanalyse: Eine Einführung für Forschung und Praxis. VS Verlag für Sozialwissenschaften, Wiesbaden

35. Lampert T, List S (2011) Tabak - Zahlen und Fakten zum Konsum. In: (DHS) DHfSeV (Hrsg) Jahrbuch Sucht 2011. Neuland Verlagsgesellschaft, Geesthacht

36. Destatis (2006) Leben in Deutschland. Haushalte, Familien und Gesundheit - Ergebnisse des Mikrozensus 2005. Statistisches Bundesamt, Wiesbaden

37. RKI (Hrsg) (2009) 20 Jahre nach dem Fall der Mauer: Wie hat sich die Gesundheit in Deutschland entwickelt. Robert Koch-Institut, Berlin

38. Destatis (2006) Fragen zur Gesundheit 2005 - Körpermaße der Bevölkerung 2005. Statistisches Bundesamt, Wiesbaden

39. Destatis (2011) Fragen zur Gesundheit 2009 - Körpermaße der Bevölkerung 2009. Statistisches Bundesamt, Wiesbaden

40. StataCorp (2009) Stata Statistical Software: Release 11.0. Stata Corporation, College Station, TX

41. Elliott P, Wartenberg D (2004) Spatial epidemiology: current approaches and future challenges. Environ Health Perspect 112:998-1006

\section{Gründung der Arbeitsgruppe Geriatrie der Deutschen Gesell- schaft für Schlafforschung und Schlafmedizin (DGSM)}

Nach zwei Jahren Lobbyarbeit bei den Gesellschaftsverantwortlichen und einem Jahr Gründungsphase stimmte die Jahreshauptversammlung der DGSM am 10. November einstimmig für die Gründung einer Arbeitsgruppe Geriatrie. Als AG Sprecher wurden die Initiatoren der AG, PD Dr. Nikolaus Netzer, Schlafmediziner und Geriater aus Bad Aibling und als sein Stellvertreter Dr. Helmut Frohnhofen, Geriater und Schlafmediziner aus Essen vom Plenum gewählt.

Diese AG Neugründung war nicht selbstverständlich, da die DGSM als Fachgesellschaft mit über 2000 Mitgliedern eigentlich die Zahl der Arbeitsgruppen verkleinern wollte und einige Arbeitsgruppen fusionieren möchte. Aber das Thema geriatrische Schlafmedizin gewann über die letzten zwei Jahre doch soviel Aufmerksamkeit und die deutschen Schlafmediziner sahen es als so wichtig an, dass hier noch einmal eine Ausnahme vom Konsolidierungskurs gemacht wurde.

Bereits im Vorfeld bei der Jahrestagung der Deutschen Gesellschaft für Geriatrie (DGG) in Bad Bramstedt hatten die Vorstandsmitglieder der DGG, Präsident PD Dr. Werner Hofmann und der kommende Präsident Prof. Dr. Ralf - Joachim Schulz erklärt, dass man sich von Seiten der DGG eine gemeinsame AG Geriatrische Schlafmedizin mit der DGSM vorstellen kann, auf Seiten der DGG könnte hier eine Fusionierung mit der AG Pneumologie der DGG stattfinden. Dieser Vorschlag wurde von den AG Teilnehmern bei der Jahrestagung der DGSM in Mannheim positiv aufgenommen. Als Beispiel für eine solche gesellschaftsübergeifende Arbeitsgruppe in der Schlafmedizin könnte die AG Apnoe dienen, die eng mit der schlafmedizinischen Arbeitsgruppe der Deutschen Gesellschaft für Pneumologie (SNAK) verwoben ist.
Die wichtigsten Ziele der neuen Arbeitsgruppe, das war bereits in vorbereitenden Treffen besprochen worden, ist die Erarbeitung spezifischer Leitlinien für hochbetagte Patienten in Schlaflaboren, und Altersgruppen spezifischer Fragebögen sowie die Veröffentlichung eines Buches zum Thema geriatrische Schlafmedizin mit deutschen und internationalen Autoren. Einen breiten Raum wird in den nächsten Jahren auch die Lobbyarbeit bei den Krankenkassen für eine faire Kostenerstattung bei den arbeitsintensiven geriatrischen Patienten im Schlaflabor einnehmen.

Nikolaus Netzer (Bad Aibling) 